\title{
A multiclass cell transmission model for shared human and autonomous vehicle roads
}

\author{
Michael W. Levin \\ Graduate Research Assistant \\ Stephen D. Boyles \\ Assistant Professor
}

July 10, 2015

\begin{abstract}
Autonomous vehicles have the potential to improve link and intersection traffic behavior. Computer reaction times may admit reduced following headways and increase capacity and backwards wave speed. The degree of these improvements will depend on the proportion of autonomous vehicles in the network. To model arbitrary shared road scenarios, we develop a multiclass cell transmission model that admits variations in capacity and backwards wave speed in response to class proportions within each cell. The multiclass cell transmission model is shown to be consistent with the hydrodynamic theory. This paper then develops a car following model incorporating driver reaction time to predict capacity and backwards wave speed for multiclass scenarios. For intersection modeling, we adapt the legacy early method for intelligent traffic management (Bento et al., 2013) to general simulation-based dynamic traffic assignment models. Empirical results on a city network show that intersection controls are a major bottleneck in the model, and that legacy early method improves over traffic signals when the autonomous vehicle proportion is sufficiently high.
\end{abstract}

Keywords: autonomous vehicles, dynamic traffic assignment, cell transmission model, multiclass, shared roads

\section{Introduction}

Autonomous vehicle (AV) technology is rapidly maturing with testing permitted on public roads in several states. When AVs become available to the public, computer precision and communications may allow new behaviors to increase network capacity. For instance, Dresner \& Stone (2004) proposed the tile-based reservation (TBR) intersection policy which 
reduces delay beyond optimized traffic signals (Fajardo et al., 2011). Besides offering new intersection behaviors, AVs may also increase link capacity because reduced reaction times requires smaller following distances, and AVs may be less affected than human-driven vehicles (HVs) by certain adverse road conditions. However, capacity improvements are complicated by sharing roads with HVs, which will likely be the case for many years before AVs are sufficiently available and affordable to be driven by all travelers.

TBR is compatible with shared roads (Dresner \& Stone, 2007), and link behaviors may be performed safely with a mixed fleet of vehicles. However, modeling link and intersection capacity improvements from shared road policies is still an open problem. Most current models of AVs are micro-simulations, which are not computationally tractable for the traffic assignment typically used to determine route choice. Levin \& Boyles (2015a) modified static link performance functions model to predict capacity improvements as a function of the proportion of AVs on each link based on Greenshields' (1935) capacity model. However, in reality the proportion of AVs on each link will vary over time. Dynamic traffic assignment (DTA) models flow more accurately than static models and can include the varying-time effects of capacity. Kesting et al. (2010) predicted theoretical capacity for adaptive cruise control and use linear regression to extrapolate for various proportions of connected vehicles $(\mathrm{CVs})$ and non-CVs. For consistency with DTA, we use a constant acceleration model to analytically predict capacity and wave speed as a function of the proportion of each vehicle class on the road, and generalize to multiple classes with different reaction times. Whereas many previous papers on CVs use micro-simulation experiments, we use DTA on a city network to study the impacts of AVs under dynamic user equilibrium (DUE) route choice.

This paper makes several contributions with the aim of developing a shared road DTA model: First, a multiclass cell transmission model (CTM) is proposed that admits space-time variations of capacity and wave speed. Second, a link capacity model based on a collision avoidance car following model with different reaction times is presented. The link capacity assumptions lead to the triangular fundamental diagram assumed by Newell (1993) and Yperman et al. (2005). To facilitate shared intersections, the conflict region (CR) algorithm from Levin \& Boyles (2015b) for general SBDTA models is modified using Bento et al. (2013)s control policy. Intersection efficiency scales dynamically with the proportion of AVs using the intersection. Results from studies on a single intersection and the downtown Austin city network suggest that travel time reductions when using reservation-based controls scale linearly with the proportion of AVs, but do not improve over signals until 80\% AV penetration or greater.

The remainder of this paper is organized as follows. Section 2 discusses literature relevant to multiclass DTA and AV flow. Section 3 presents the multiclass DTA model and shows consistency with the hydrodynamic theory of traffic flow. Section 4 develops a dynamic capacity and wave speed model based on driver reaction times. A shared intersection model for general SBDTA is developed in Section 5. In Section 6, we present a case study on a city network involving varying levels of human-driven and autonomous vehicles, and Section 7 discusses conclusions. 


\section{Literature review}

This literature review starts by discussing multiclass DTA in Section 2.1 to provide a context for the AV models discussed in Section 2.2.

\subsection{Dynamic traffic assignment}

DTA includes a number of different flow models, some of which are solved analytically and others which are simulation-based (SBDTA). For an overview of DTA, we refer to Chiu et al. (2011). This paper focuses on the cell transmission (CTM) SBDTA model (Daganzo, 1994; 1995a), which is a discrete approximation of the Lighthill-Whitham-Richards (LWR) model (Lighthill \& Whitham, 1955; Whitham, 1956). The partial differential equations of the LWR model are generally more difficult to solve when multiple vehicle classes result in varying capacities. However, the discretized space and time in CTM simplifies the multiclass solution method. The multiclass CTM presented in Section 3 is shown to be compatible with the conservation equations of LWR.

Multiclass DTA has previously been studied in the literature although primarily with a focus on heterogeneous vehicles of length and speed. Most previous studies have been concerned with speed differences among different types of vehicles, such as passenger cars and heavy trucks. Hoogendorn \& Bovy (2001) developed a platoon-based multiclass theory along with a cell-based discretization. Although limited to two classes, Chanut \& Buisson (2003) accounted for differences in speeds and lengths and created a Godunov scheme for simulation. Logghe \& Immers (2008) developed a multiclass kinematic wave theory to study how slowmoving vehicle classes can become moving bottlenecks for faster-moving vehicles. They also use a fundamental diagram varying with class densities and suggest a cell discretization. van Wageningen-Kessels et al. (2014) studied a kinematic wave model to determine how the proportion of classes affects congestion as flow increases, specifically considering the greater length and following headways necessary for heavy trucks. Wong \& Wong (2002) allowed vehicles to have a class-specific speed and demonstrate that their model adheres to flow conservation. However, they use a new discrete space-time approximation to solve their model, and it is not clear whether it is compatible with the most common simulation-based approximations, which is desirable for integration with existing DTA models. Tuerprasert \& Aswakul (2010) formulated a multiclass CTM with different speeds per class, including how different speeds affect cell propagation. It is not clear, though, whether their model solves a multiclass form of LWR, or is a modification of CTM with useful properties. Overall, models for multiclass traffic flow have been extensively studied to model vehicle types with different speeds. This is somewhat orthogonal to our objective, and considerably more difficult to solve. The purpose of the multiclass CTM presented in Section 3 of this paper is to model a fundamental diagram changing in response to reaction times rather than vehicle speeds. We also use CTM to be tractable for DTA on large networks and compatible with existing simulators. 


\subsection{Autonomous vehicle flow}

The model presented in this paper is concerned with varying capacities and wave speeds due to the multiple classes of human-driven and autonomous vehicles. We assume that speed does not depend on vehicle class, which is reasonable because some AVs are programmed to exceed the speed limit to maintain the same speed as surrounding traffic (Miller, 2014) for improved safety (Aarts \& Van Schagen, 2006).

Potential improvements in traffic flow from CVs and $\mathrm{AVs}$ have begun to receive attention in the literature. Adaptive cruise control (ACC) (Marsden et al., 2001) has been developed to improve link capacity and, if it is not incorporated into AVs, will likely influence AV carfollowing behavior. Nilsson et al. (2014) develop initial correct-by-construction algorithms for ACC to advance its implementation on roads. To study the effects of ACC on freeway traffic, many studies have developed micro-simulation models. Van Arem et al. (2006) used a micro-simulation to show that cooperative ACC can improve efficiency. Kesting et al. (2010) developed a continuous acceleration behavior model of CVs to predict theoretical capacity. They use a linear regression to extrapolate for different proportions of CVs and non-CVs. We generalize by including multiple vehicle classes with different reaction times in our constant acceleration model and predict both capacity and wave speed as a function of the proportion of each vehicle class. Li \& Shrivastava (2002) proposed using adaptive cruise control for a constant time headway policy on freeways resulting in stable flow when merge/diverges were suitably controlled. Schakel et al. (2010) used simulation to study traffic flow stability, finding that ACC increases stability and also increases shockwave speed. This is consistent with the theoretical fundamental diagram we develop in Section 4. Although much of the literature uses micro-simulation to study CVs and AVs, we use the predicted capacities and wave speeds in a DTA model to study the impacts on a city network with DUE.

A major topic in the literature is new intersection policies for AVs. Dresner \& Stone (2004) developed a reservation-based policy (TBR) using the greater precision and more complex communications possible with AVs. Fajardo et al., (2011) found that TBR improved over optimized traffic signals. Because TBR subsumes traffic signals, signals can be combined with an intersection agent controller to make TBR compatible with shared roads through an alternate reservation-granting policy (Dresner and Stone, 2007). Bento et al. (2013) proposed to extend TBR to non-communication equipped vehicles by reserving additional space to account for reduced precision and unknown destination, and Qian et al. (2014) developed a provably collision-free shared-intersection system. Other reservation prioritization policies with the goal of reducing intersection delay have been explored, such as intersection auctions (Schepperle \& Bhm, 2007; Vasirani \& Ossowski, 2012; Carlino et al., 2013). Analyzing TBR on city-size networks has been a major challenge as most AV traffic models have used micro-simulation. Carlino et al. (2012) used a simplified non-tile-based reservation policy to simulate a large network in reasonable time. However, the intersection capacity of this model was significantly reduced. Because of the number of simulations involved in solving DTA for user equilibrium, a micro-simulation model of intersections is not sufficient. Levin \& Boyles (2015b) used a conflict region (CR) simplification to make TBR computationally tractable for DTA, and an extension of the CR model is used for 


\section{Multiclass cell transmission model}

This section presents a multiclass extension of CTM. The focus of this paper is on roads with both human and autonomous personal vehicles. Vehicles differ in the driver behavior but not in the physical performance characteristics. Therefore, we do not include the speed differences between vehicle classes, such as between heavy trucks and personal vehicles. The models in Sections 3 and 4 are defined for continuous flows, which some DTA models use. Because this paper is also concerned with node models, and because reservation-based intersection controls are defined for discrete vehicles, Sections 5 and 6 will discretize the flow model defined here. In this paper, we make the following assumptions.

1. All vehicles travel at the same speed. Although in reality vehicle speeds differ, in DTA models the vehicle speed behavior model is often assumed to be identical for all vehicles. This is reasonable even with multiple vehicle classes because AVs may match the speed of surrounding vehicles even if it requires exceeding the speed limit (Miller, 2014). Although Tuerprasert \& Aswakul (2010) consider different vehicle speeds in CTM, in this study of HVs and AVs much of the differences in speed would come from variations in $\mathrm{HV}$ behavior that are often not considered in DTA models.

2. Uniform distribution of class-specific density per cell. Single-class CTM assumes the density within a cell is uniformly distributed. We extend that assumption to classspecific densities.

3. Arbitrary number of vehicle classes. Although this study focuses on the transition from HVs to AVs, different types of AVs may be certified for different reaction times, and thus may respond differently in their car-following behavior.

4. Backwards wave speed is less than or equal to free flow speed. This is necessary to determine cell length by free flow speed. Although this is a common assumption in DTA models, in Section 4 we show that a sufficiently low reaction time might break this assumption.

We first define the multiclass hydrodynamic theory in Section 3.1. Then, following the presentation of Daganzo (1994), we state the cell transition equations in Section 3.2 and show that they are consistent with the multiclass hydrodynamic theory in Section 3.3.

\subsection{Multiclass hydrodynamic theory}

Let $M$ be the set of vehicle classes. Let $k_{m}(x, t)$ be the density of vehicles of class $m$ at space-time point $(x, t)$ with total density denoted by $k(x, t)=\sum_{m \in M} k_{m}(x, t)$. Similarly, let $q_{m}(x, t)=u\left(\frac{k_{1}}{k}, \ldots, \frac{k_{|M|}}{k}\right) k_{m}(x, t)$ be the class-specific flow, with the total flow given by 
$q(x, t)=\sum_{m \in M} q_{m}(x, t)$, and let the function $u\left(\frac{k_{1}}{k}, \ldots, \frac{k_{|M|}}{k}\right)$ denote the speed possible with class proportions of $\frac{k_{1}}{k}, \ldots, \frac{k_{|M|}}{k}$.

Speed is limited by free flow speed, capacity, and backwards wave propagation:

$$
u\left(k_{1}, \ldots k_{|M|}\right)=\min \left\{u^{\mathrm{f}}, \frac{q^{\max }\left(\frac{k_{1}}{k}, \ldots, \frac{k_{|M|}}{k}\right)}{k}, w\left(\frac{k_{1}}{k}, \ldots, \frac{k_{|M|}}{k}\right)\left(k^{\mathrm{jam}}-k\right)\right\}
$$

where $u^{\mathrm{f}}$ is free flow speed, $w\left(\frac{k_{1}}{k}, \ldots, \frac{k_{|M|}}{k}\right)$ is the backwards wave speed, $q^{\max }\left(\frac{k_{1}}{k}, \ldots, \frac{k_{|M|}}{k}\right)$ is the capacity when the proportions of density in each class are $\frac{k_{1}}{k}, \ldots, \frac{k_{|M|}}{k}$, and $k^{\mathrm{jam}}$ is jam density. $k^{\mathrm{jam}}$ is assumed not to depend on vehicle type, as the physical characteristics (such as length and maximum acceleration) of human-driven and autonomous vehicles are assumed to be the same. For consistency, conservation of flow must be satisfied, i.e. $\frac{\partial q_{m}(x, t)}{\partial x}=-\frac{\partial k_{m}(x, t)}{\partial t}$ for all $m \in M$ (Wong and Wong, 2012).

\subsection{Cell transition flows}

As with Daganzo (1994), to form the multiclass CTM we discretize time into time steps of $d t$. Links are then discretized into cells labeled by $i=1, I$ such that vehicles traveling at free flow speed will travel exactly the distance of one cell per time step. Let $n_{i}^{m}(t)$ be vehicles of class $m$ in cell $i$ at time $t$, where $n_{i}(t)=\sum_{m \in M} n_{i}^{m}(t)$. Let $y_{i}^{m}(t)$ be vehicles of class $m$ entering cell $i$ from cell $i-1$ at time $t$. Then cell occupancy is defined by

$$
n_{i}^{m}(t+1)=n_{i}^{m}(t)+y_{i}^{m}(t)-y_{i+1}^{m}(t)
$$

with total transition flows given by

$$
y_{i}(t)=\sum_{m \in M} y_{i}^{m}(t)=\min \left\{\sum_{m \in M} n_{i-1}^{m}(t), Q_{i}(t), \frac{w_{i}(t)}{u^{\mathrm{f}}}\left(N-\sum_{m \in M} n_{i}^{m}(t)\right)\right\}
$$

where $N$ is the maximum number of vehicles that can fit in cell $i$ and $Q_{i}(t)$ is the maximum flow.

Equation (3) defines the total transition flows, which will now be defined specific to vehicle class. To avoid dividing by zero, assume $n_{(i-1)}(t)>0$. (If $\left.n_{(} i-1\right)(t)=0$, there is no flow to propagate). As stated in Assumption 2, class-specific density is assumed to be uniformly distributed throughout the cell. Then class-specific transition flows are proportional to $\frac{n_{i-1}^{m}(t)}{n_{i-1}(t)}$ :

$$
y_{i}^{m}(t)=\frac{n_{i-1}^{m}(t)}{n_{i-1}(t)} \min \left\{\sum_{m \in M} n_{i-1}^{m}(t), Q_{i}(t), \frac{w_{i}(t)}{u^{\mathrm{f}}}\left(N-\sum_{m \in M} n_{i}^{m}(t)\right)\right\}
$$




$$
y_{i}^{m}(t)=\min \left\{n_{i-1}^{m}(t), \frac{n_{i-1}^{m}(t)}{n_{i-1}(t)} Q_{i}(t), \frac{n_{i-1}^{m}(t)}{n_{i-1}(t)} \frac{w_{i}(t)}{u^{\mathrm{f}}}\left(N-\sum_{m \in M} n_{i}^{m}(t)\right)\right\}
$$

which shows that flow of class $m$ is restricted by three factors: 1) class-specific cell occupancy; 2) proportional share of the capacity; and 3) proportional share of congested flow.

In the general hydrodynamic theory, class proportions may vary arbitrarily with space and time, which includes the possibility of variations within a cell. Therefore, assuming uniformly distributed density results in the possibility of non-FIFO behavior within cells. One class may have a higher proportion at the end of the cell, and thus might be expected to comprise a higher proportion of the transition flow. However, as discussed by Blumberg \& Bar-Gera (2009), even single class CTMs may violate FIFO. The numerical experiments in this paper use discretized flow to admit reservation-based intersection models. The discretized flow also allows vehicles within a cell to be contained within a FIFO queue, which ensures FIFO behavior at the cell level. Total transition flows for discrete vehicles are determined as stated above for continuous flow.

\subsection{Consistency with hydrodynamic theory}

As with Daganzo (1994) we show that these transition flows are consistent with the multiclass hydrodynamic theory defined in Section 3.1. Assume class-specific flow is proportional to density, i.e. $\frac{k_{m}}{k}$, and all classes travel at the same speed. Also assume that $k>0$, because if $k=0$ then flow is also 0 . Then

$$
q_{m}(x, t)=\frac{k_{m}}{k} \min \left\{u^{\mathrm{f}} k, q^{\max }\left(\frac{k_{1}}{k}, \ldots, \frac{k_{|M|}}{k}\right), w k\left(\frac{k_{1}}{k}, \ldots, \frac{k_{|M|}}{k}\right)\left(k^{\mathrm{jam}}-k\right)\right\}
$$

Let $d t$ be the time step and choose cell length such that $u^{\mathrm{f}} \cdot d t=1$. Then cell length is $1, u^{\mathrm{f}}$ is $1, x=i, k^{\mathrm{jam}}=N, q^{\max }(t)=Q(t)$, and $k(x, t)=n_{i}(t)$. Cell length is chosen so that flow may traverse at most one cell per time step to satisfy the Courant-Friedrichs-Lewy conditions (Courant et al., 1928). Then

$$
q_{m}(x, t)=\frac{n_{i}^{m}(t)}{n_{i}(t)} \min \left\{n_{i}(t), q_{i}^{\max }(t), \frac{w_{i}(t)}{v}\left(N-n_{i}(t)\right)\right\}=y_{i+1}^{m}(t)
$$

except for the subindex of $n$ the last term, which should be $i+1$. As with Daganzo (1994) this difference is disregarded. (See Daganzo, 1995b for more discussion on this issue.) Therefore $\frac{\partial q_{m}(x, t)}{\partial x}=y_{i+1}^{m}(t)-y_{i}^{m}(t)$. Since $\frac{\partial k_{m}(x, t)}{\partial t}=n_{i}^{m}(t+1)-n_{i}^{m}(t)$ is the rate of change in cell occupancy with respect to time, the conservation of flow equation $\frac{\partial q_{m}(x, t)}{\partial x}=-\frac{\partial k_{m}(x, t)}{\partial t}$ is satisfied by the cell propagation function of equation (2). 
We now present a car following model based on kinematics to predict the speed-density relationship as a function of the reaction times of multiple classes. Car following models can be divided into several types as described by Brackstone et al. (1999) and Gartner et al. (2005). For instance, some predict fluctuations in the acceleration behavior of an individual driver in response to the vehicle ahead. However, for DTA a simpler model is more appropriate to predict the speed of traffic at a macroscopic level. Newell (2002) greatly simplified car following to be consistent with the hydrodynamic theory, but the model does not include the effects of reaction time. Instead, the car following model used here builds from the collision avoidance theory of Kometani \& Sasaki (1959) to predict the allowed headway for a given speed, which varies with driver reaction time. The inverse relationship predicts speed as a function of the headway, which is determined by density. This car following model results in the triangular fundamental diagram used by Newell (1993) and Yperman et al. (2005).

Although this car following model is useful in predicting the effects of a heterogeneous vehicle composition on capacity and wave speed, other effects such as roadway conditions are not included. Furthermore, CTM assumes a trapezoidal fundamental diagram that admits a lower restriction on capacity. Therefore, the effect of reaction times on capacity and backwards wave speed are used to appropriately scale link characteristics for realistic city network models. Although AVs may be less affected by adverse roadway conditions than human drivers, this paper assumes similar effects for the purposes of developing a DTA model of shared roads. Other estimations of capacity and wave speed may also be included in the multiclass CTM model developed in Section 3.

\subsection{Safe following distance}

Suppose that vehicle 2 follows vehicle 1 at speed $u$ with vehicle lengths $\ell$. Vehicle 1 decelerates at $a$ to a full stop starting at time $t=0$, and vehicle 2 follows suit after a reaction time of $\Delta t$. The safe following distance, $L$, is determined by kinematics.

The position of vehicle 1 is given by

$$
x_{1}(t)= \begin{cases}u t-\frac{1}{2} a t^{2} & t \leq \frac{u}{a} \\ \frac{u^{2}}{2 a} & t>\frac{u}{a}\end{cases}
$$

where $\frac{u}{a}$ is the time required to reach a full stop. For $t>\frac{u}{a}$, the position of vehicle 1 is constant after its full stop. The position of vehicle 2 , including the following distance of $L$, is

$$
x_{2}(t)= \begin{cases}u t-L & t \leq \Delta t \\ u t-\frac{1}{2} a(t-\Delta t)^{2}-L & t>\Delta t\end{cases}
$$


The difference is

$$
x_{1}(t)-x_{2}(t)= \begin{cases}u-\frac{1}{2} a t^{2}+L & t \leq \Delta t \\ -a t \Delta t+\frac{1}{2} a(\Delta t)^{2}+L & \Delta t<t \leq \frac{u}{a} \\ \frac{u^{2}}{2 a}-u t+\frac{1}{2} a(t-\Delta t)^{2}+L & t>\frac{u}{a}\end{cases}
$$

and the minimum distance occurs when both vehicles are stopped, at $\frac{u}{a}+\Delta t$. To avoid a collision,

$$
L \geq-\frac{u^{2}}{2 a}+u\left(\frac{u}{a}+\Delta t\right)-\frac{1}{2} a\left(\frac{u}{a}\right)^{2}+\ell=u \Delta t+\ell
$$

\subsection{Flow-density relationship}

Equivalently, equation (11) may be expressed as

$$
u \leq \frac{L-\ell}{\Delta t}
$$

which restricts speed based on following distance (from density). Flow may be determined from the relationship $q=\left(\frac{L-\ell}{\Delta t}\right) k$ with $L=\frac{1}{k}$, which is linear with respect to density. Figure 1 shows the resulting relationship between flow and density for different reaction times for a characteristic vehicle of length $20 \mathrm{ft}(6.1 \mathrm{~m})$ that decelerates at $9 \mathrm{ft} / \mathrm{s}^{2}(2.7$ $\left.\mathrm{m} / \mathrm{s}^{2}\right)$ for a free flow speed of $60 \mathrm{mi} / \mathrm{hr}(96.6 \mathrm{~km} / \mathrm{hr})$. Since speed is bounded by free flow speed and available following distance, the triangular fundamental diagram is described by

$q=\min \left\{u^{\mathrm{f}} k,\left(\frac{L-\ell}{\Delta t}\right) k\right\}$. Reaction times of 1 to 1.5 seconds correspond to human drivers (Johansson \& Rumar, 1971).

The maximum density at which a speed of $u$ is possible is $\frac{1}{u \Delta t+\ell}$ from equation (12), and therefore capacity for free flow speed of $u^{f}$ is

$$
q^{\max }=u^{\mathrm{f}} \frac{1}{u^{\mathrm{f}} \Delta t+\ell}
$$

Backwards wave speed is

$$
w=-\frac{\frac{u^{\mathrm{f}}}{u^{\mathrm{f}} \Delta t+\ell}}{\frac{1}{u^{\mathrm{f}} \Delta t+\ell}-\frac{1}{\ell}}=\frac{\ell}{\Delta t}
$$

which increases as reaction time decreases. The direction of this relationship is consistent with micro-simulation results by Schakel et al. (2010). Note that if $\Delta t<\frac{\ell}{u^{f}}$, which may be possible for computer reaction times, then backwards wave speed exceeds free flow speed. If $w>u^{\mathrm{f}}$ for CTM, then the cell lengths would need to be derived from the backward wave speed, not the forward. That would complicate the cell transition flows. To avoid this issue, this paper assumes that $w \leq u^{\mathrm{f}}$. 


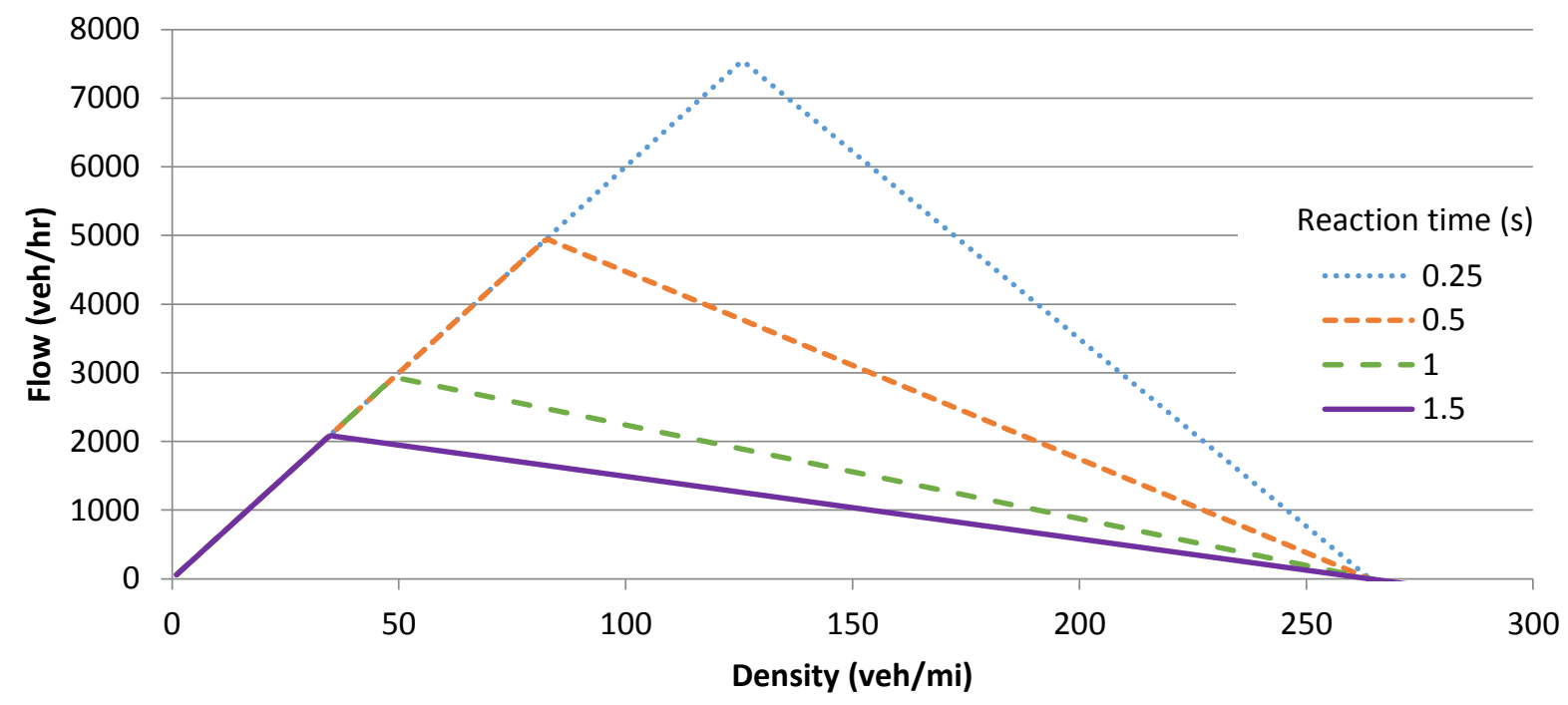

Figure 1: Flow-density relationship as a function of reaction time

\subsection{Flow for heterogeneous vehicles}

The car following model in Section 4.2 is designed to estimate the capacity and backwards wave speed when the reaction time varies, but is uniform across all vehicles. This section expands the model for heterogeneous flow with different vehicles having different reaction times. Let the density be disaggregated into $k_{m}$ for each vehicle class $m$. Consider the case where speed is limited by density. Assuming that all vehicles travel at the same speed, for all vehicle classes,

$$
u=\frac{L_{m}-\ell}{\Delta t_{m}}
$$

where $L_{m}$ is the headway allotted and $\Delta t_{m}$ is the reaction time for vehicles of class $m$. Also, with appropriate units,

$$
\sum_{m \in M} k_{m} L_{m}=1
$$

is the total distance occupied by the vehicles. Thus

$$
\sum_{m \in M} k_{m}\left(L_{m}-\ell\right)=1-k \ell
$$

By equation (15),

$$
\sum_{m \in M} k_{m} u \Delta t_{m}=1-k \ell
$$

which results in

$$
u=\frac{1-k \ell}{\sum_{m \in M} k_{m} \Delta t_{m}}
$$


Equations (19) through (23) reduce to the model in Section 4.2 in the single vehicle class scenario. Figure 2 shows an example of how capacity and wave speed increase as the AV proportion increases when human drivers have a reaction time of 1 second and autonomous vehicles have a reaction time of 0.5 second. The cases of $0 \%$ AVs and $100 \%$ AVs are identical to the 1 second reaction time and 0.5 second reaction time fundamental diagrams in Figure 1 , respectively.

\subsection{Other factors affecting capacity}

In reality, factors such as narrow lanes and road conditions affect capacity as well. These factors are usually in Highway Capacity Manual estimates of roadway capacity used for city network models. The model above, however, does not include factors beyond speed limit. To include these factors in the experimental results in Section 5, we scale existing estimates on capacity and wave speed in accordance with equations (22) and (23). Although the model in Section 4.3 predicts a triangular fundamental diagram as used by Newell (1993) and Yperman et al. (2005), other flow-density relationships are often used. CTM, the basis for multiclass DTA in this paper, uses a trapezoidal fundamental diagram.

Assume estimated roadway capacity and wave speed are $\hat{q}^{\max }$ and $\hat{w}$, respectively, and that the reaction time for human drivers is $\Delta t_{\mathrm{HV}}$. Human reaction times may vary depending on the location of the road; for instance reaction times on rural roads are often greater than 


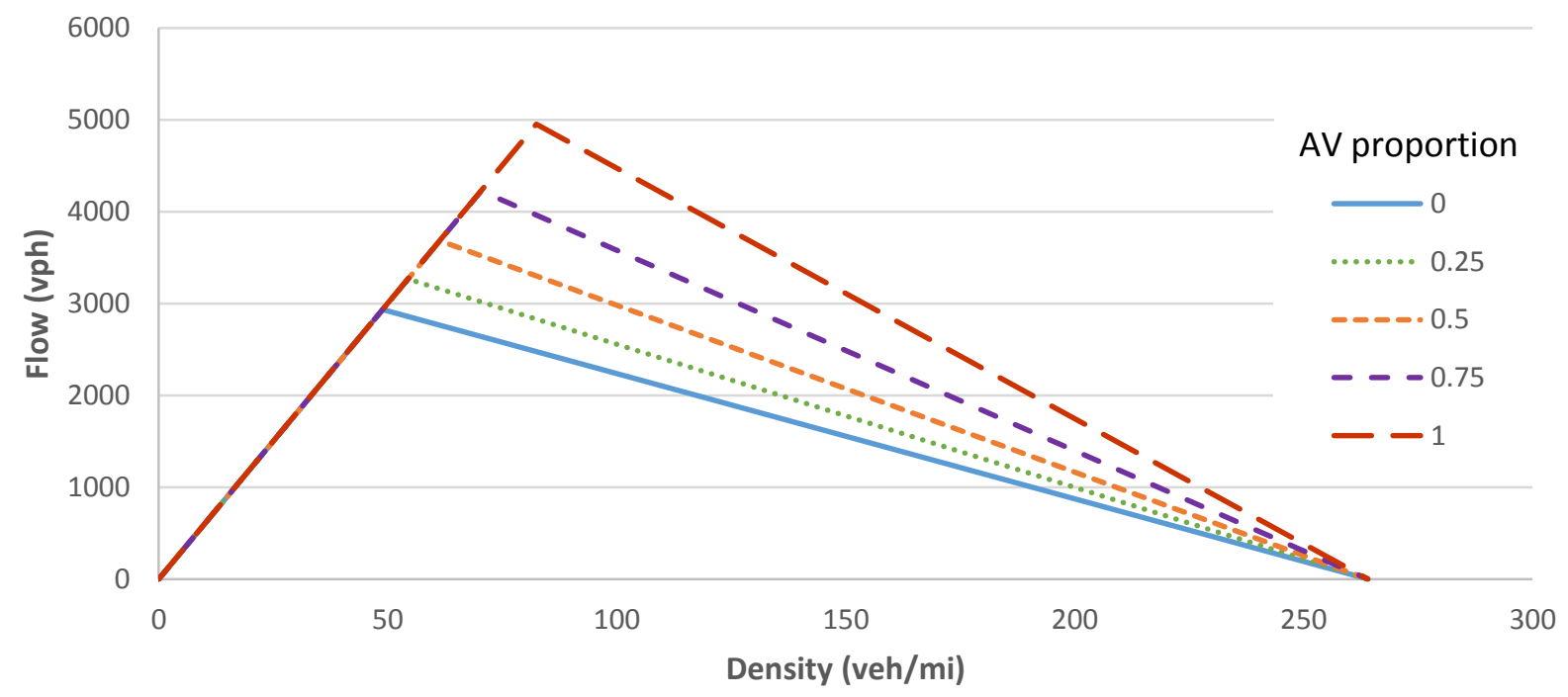

Figure 2: Flow-density relationship as a function of AV proportion.

those in the city. Because capacity is affected by reaction time through equation (22), scaled capacity $\tilde{q}^{\max }$ is

$$
\tilde{q}^{\max }=\frac{u^{\mathrm{f}} \Delta t_{\mathrm{HV}}+\ell}{u^{\mathrm{f}} \sum_{m \in M} \frac{k_{m}}{k} \Delta t_{m}+\ell} \hat{q}^{\max }
$$

Similarly, wave speed is affected by reaction time through equation (23), so scaled wave speed $\tilde{w}$ is

$$
\tilde{w}=\frac{\Delta t_{\mathrm{HV}}}{\sum_{m \in M} \frac{k_{m}}{k} \Delta t_{m}} \hat{w}
$$

Equations (24) and (25) provide a method to integrate the capacity and backwards wave speed scaling of Section 4.3 with other factors and realistic data.

\section{$5 \quad$ Intersection control policy}

For shared road models, the intersection control policy is an important question. With 100\% human vehicles, optimized traffic signals are the best option available. With $100 \%$ AVs, TBR can reduce delay beyond that of optimized signals (Fajardo et al., 2011). The difficulty is the choice of intersection control policy for shared roads. Dresner \& Stone (2007) show that TBR subsumes traffic signals because the signal essentially reserves parts of the intersection. They propose link- and lane-cycling signals, where each link or lane successively receives full access to the intersection, and vehicles in other links or lanes may reserve non-conflicting paths. However, blocking out large portions of the intersection for a signal greatly restricts reservations from other links due to the possibility of conflict, even when most vehicles are 

large. It is also an open question whether link- or lane-cycling signals even outperform optimized traffic signals.

Bento et al. (2013) propose the legacy early method for intelligent traffic management (LEMITM) policy of reserving space-time for all possible turning movements and increasing the safety margins for non-AVs to allow them to use the TBR infrastructure. AVs still use conventional TBR, reserving only the requested path. This may be less efficient than traffic signals at small proportions of AVs because of the extra space-time reserved to ensure safety. However, as the proportion of AVs increases, TBR/LEMITM will devote less space-time to safety of human vehicles because it is not constrained by protecting turning movements allowed by traffic signals. As a result, TBR/LEMITM may scale at a higher rate. Therefore, TBR/LEMITM is used in this paper to study how link and intersection capacity scales with the proportion of AVs.

TBR/LEMITM makes two assumptions that we elaborate on here for the purposes of describing the DTA model of TBR/LEMITM. First, it separates vehicles into two groups: those that can establish digital communications on reservation acceptance and adherence, and those that cannot. The latter group consists of all non-AVs, although some AVs could conceivably fall into that group as well. This is possible in practice because current technology can already determine whether a vehicle is waiting at the intersection for actuated signals. Given that a vehicle is waiting, the intersection controller need only check whether the vehicle has established digital communications, which can be determined if vehicles transmit their position to the intersection controller along with reservation requests. Second, due to the unpredictably of human behavior, the intersection controller must be able to cancel granted reservations for AVs if a human is delayed in reacting to permission to enter the intersection. Because this DTA model does not include potential human errors and takes a more aggregate view of the intersection, canceled reservations are not included in the model.

Most studies on reservation-based controls use micro-simulation and are therefore not computationally tractable for the number of simulations required to solve DTA. Levin \& Boyles (2015b) simplify TBR using the idea of larger conflict regions (CR) to distribute intersection capacity and receiving flows to sending flows for compatibility with general SBDTA models. Although the CR model is designed for arbitrary vehicle prioritization, TBR/LEMITM requires the intersection controller to reserve additional space and therefore make additional availability checks. Section 5.1 details the modifications to the CR algorithm to accommodate TBR/LEMITM.

\subsection{Modified conflict region model}

The conflict region model is a polynomial-time algorithm performed at each intersection each time step to determine intersection movement. Vehicle movement is restricted by capacity of each conflict region it passes through during its turning movement. The purpose of the conflict region algorithm (Algorithms $1 \& 2$ ) is to determine which vehicles move subject to the constraints of sending flow, receiving flow, and conflict region capacity. The development of the conflict region algorithm is described in greater detail by Levin \& Boyles (2015b). This 
The conflict region model requires discretized flow because of the priority function. For instance, Dresner \& Stone (2004) propose a first-come-first-serve priority, and Dresner \& Stone (2006) suggest priority for emergency vehicles. Modeling such prioritization functions with continuous flow is an open question, so discretized flow is used instead. These prioritization functions are orthogonal to the TBR/LEMITM control policy, although the communications required for more complex prioritization functions such as auctions may be difficult for human drivers.

Let $\Gamma^{-1}$ be the set of incoming links and $\Gamma$ be the set of outgoing links for the intersection. The intersection is divided into a set of non-overlapping conflict regions $C$, with $C_{i j}$ the subset of $C$ through which vehicles turning from $i \in \Gamma^{-1}$ to $j \in \Gamma$ will pass. Let $y_{i j}(t)$ be the number of vehicles that have moved from $i$ to $j$ and $y_{c}$ be the equivalent flow that has entered conflict region $c$ in time step $t$. Let $Q_{i}$ be the capacity of link $i$ and $Q_{i j}=\min \left\{Q_{i}, Q_{j}\right\}$ be the capacity of the turning movement from $i$ to $j$. Every conflict region has some capacity

$$
Q_{c}=\max _{(i, j) \mid c \in C_{i j}}\left\{Q_{i j}\right\}
$$

to allow flow of $\min \left\{Q_{i}, Q_{j}\right\}$ for any $(i, j)$ such that $c \in C_{i j}$ if no other demand is present, and vehicles traveling from $i$ to $j$ consume $\frac{Q_{c}}{Q_{i j}}$ of the capacity of $c \cdot \frac{Q_{c}}{Q_{i j}}>1$ refers to the case in which a vehicle from one approach reserves a capacity equivalent to more than 1 vehicle from another approach. For example, in a local road-arterial intersection, 1 vehicle crossing the intersection from the local road might prevent 2 vehicles on the arterial from moving.

Let $l_{i}$ be the number of lanes and $S_{i}(t)$ the sending flow of link $i$ at time $t$, i.e. the set of vehicles that could leave $i$ at $\mathrm{t}$ if no other constraints were present. Each vehicle $v$ has some priority defined by the arbitrary function $f(v, i)$. Let $R_{j}(t)$ be the receiving flow of link $j$, i.e. the number of vehicles that could enter $j$ at $t$ if incoming flow was infinite. Sending and receiving flows are general characteristics of dynamic flow models and allow the CR model to be applied to general SBDTA models. Denote by $\delta_{v}^{\mathrm{AV}}$ whether vehicle $v$ is autonomous.

Two modifications to the control algorithm are required to implement TBR/LEMITM. First, for non-AVs, movement from $i$ to $j$ across the intersection requires available capacity for all possible turning movements from $i$ because the vehicle cannot communicate its destination to the intersection controller. The set of conflict regions a vehicle leaving link $i$ could pass through is $\cup_{j^{\prime} \in \Gamma} C_{i j^{\prime}}$. It is not specific to $j$ because for a human vehicle, the intersection manager does not know the vehicle's destination link. Therefore the intersection controller must check whether all such turning movements have space available. Second, when such a reservation is accepted, space for all possible turning movements from $i$ must be reserved. The modified CR model is formalized in Algorithms 1 \& 2.

\subsection{Adjusted flows for vehicle classes}

As shown in Section 4, cell capacities can be adjusted based on the reaction times of vehicles in the cell. However, CR capacities cannot similarly be adjusted because it is not known in advance which vehicles will pass through. Instead, the equivalent flow is adjusted based on 


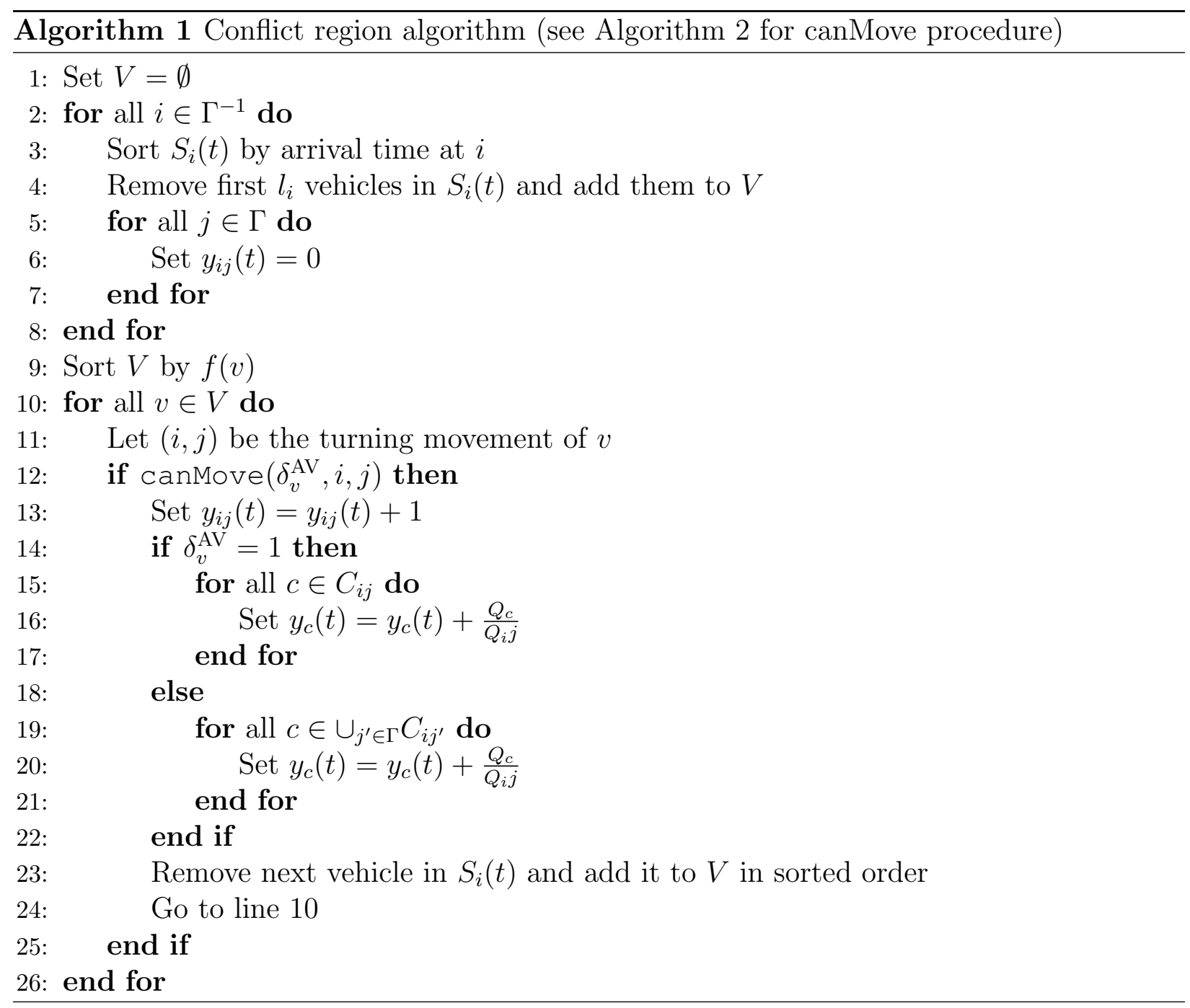




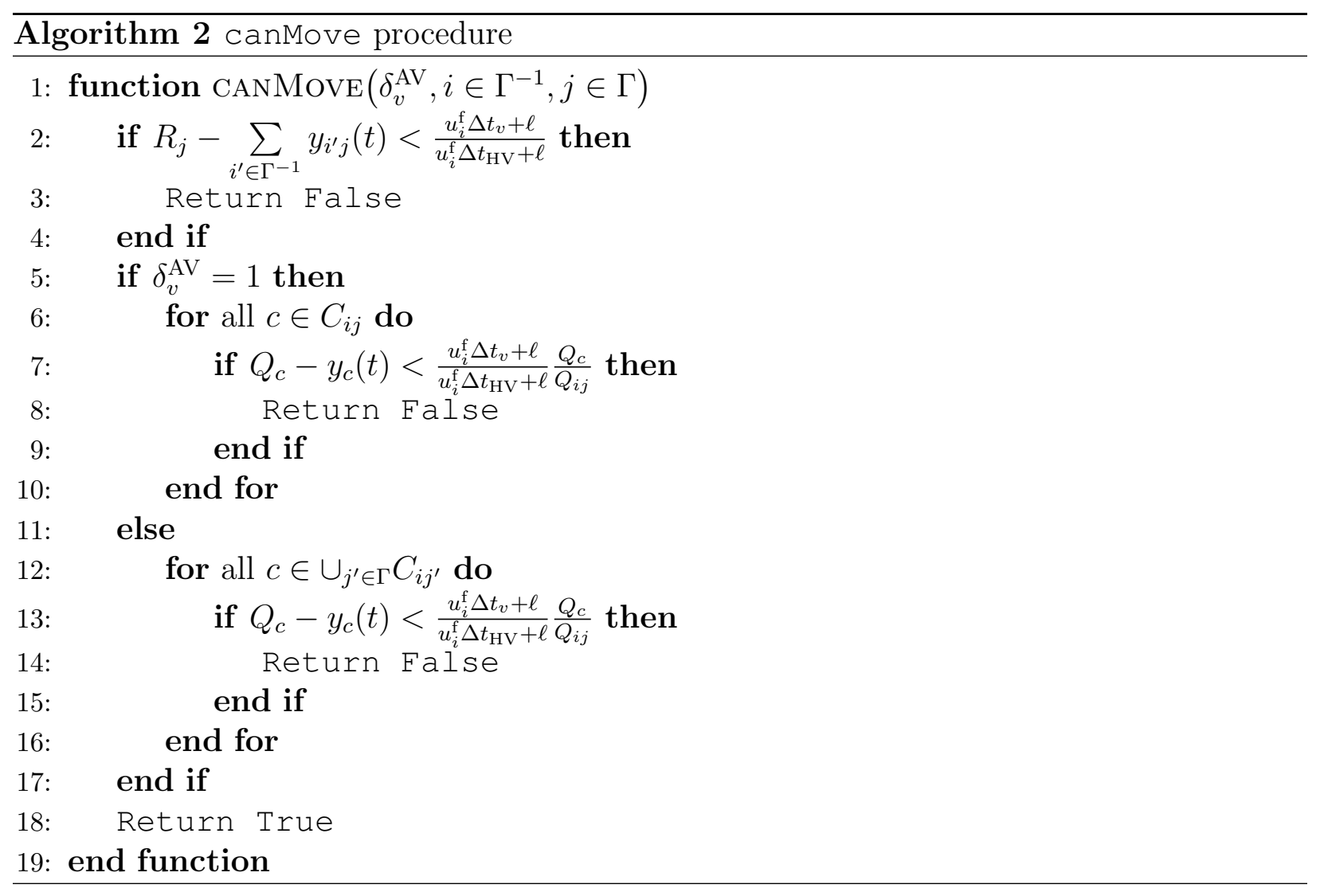


the vehicle reaction time. Levin \& Boyles (2015b) adjust the equivalent flow to account for differences in speeds from incoming links. Here we define an adjustment to equivalent flow because of differences in density due to reaction times. Vehicles with lower reaction times consume a smaller amount of the capacity. Based on equation (24), when the base capacity $\hat{q}^{\max }$ is used to determine CR capacity, a vehicle $v$ with reaction time $\Delta t_{v}$ should have an equivalent flow of

$$
\frac{u_{i}^{\mathrm{f}} \Delta t_{v}+\ell}{u_{i}^{\mathrm{f}} \Delta t_{\mathrm{HV}}+\ell}
$$

where $u_{i}^{\mathrm{f}}$ is the free flow speed of link $i$.

\section{$6 \quad$ Experimental results}

This section describes the results of two experiments using multiclass CTM and TBR/LEMITM. All experiments used a custom DTA software implemented in Java. First we study a single intersection to determine how TBR/LEMITM affects intersection delay as the proportion of AVs increases. Second, we implement the shared road model in DTA on the downtown Austin city network with varying proportions of AVs to study the effects on total travel time and compare with traffic signals. Although TBR/LEMITM was introduced by Bento et al. (2013), their experiments are focused on the efficiency of the various intersection controls they study rather than their use in combination. Therefore the experiments in this section are a first look at using TBR/LEMITM as needed in a shared road scenario. These are also the first results for shared roads with DUE routing behavior.

For these experiments, flow is discretized so reservation-based intersection controls may be used. As a result, vehicles within a cell are contained in a FIFO queue, and FIFO is ensured within cells except at intersections. Cell transition flows are restricted by capacity and cell density as functions of class proportions as discussed in Sections 3 and 4.

To study a gradual shift from HVs to AVs, flow is separated into two classes: HVs with a reaction time of 1 second, and $A V s$ with a reaction time of 0.5 seconds. $\ell$ is $20 \mathrm{ft}(6.1 \mathrm{~m})$ for the purposes of car following and jam density. The experiments hold the total demand fixed while changing the proportion of AVs. Based on equation (27), with the parameters of this study, AVs require 0.593 of the capacity that HVs require. The vehicular demand places, on average, $1400\left(p^{\mathrm{HV}}+0.593 p^{\mathrm{AV}}\right)+1300\left(p^{\mathrm{HV}}\right)$ vehicles per hour demand on the intersection in each direction.

\subsection{Single intersection}

First, we study the four link, single lane intersection shown in Figure 3 with capacity and demand chosen to demonstrate two observed conditions for the effects of TBR/LEMITM on single intersections. Each approach has demand of $1200 \mathrm{vph}$ through traffic, $200 \mathrm{vph}$ rightturning, and $100 \mathrm{vph}$ left-turning traffic. Each link is $1 \mathrm{mi}(1.6 \mathrm{~km})$ long and has capacity of $1800 \mathrm{vph}$, which does not allow all demand to be satisfied on average when a significant proportion of vehicles are HVs. Links have a free flow speed of $60 \mathrm{mi} / \mathrm{hr}(96.6 \mathrm{~km} / \mathrm{hr})$ and 


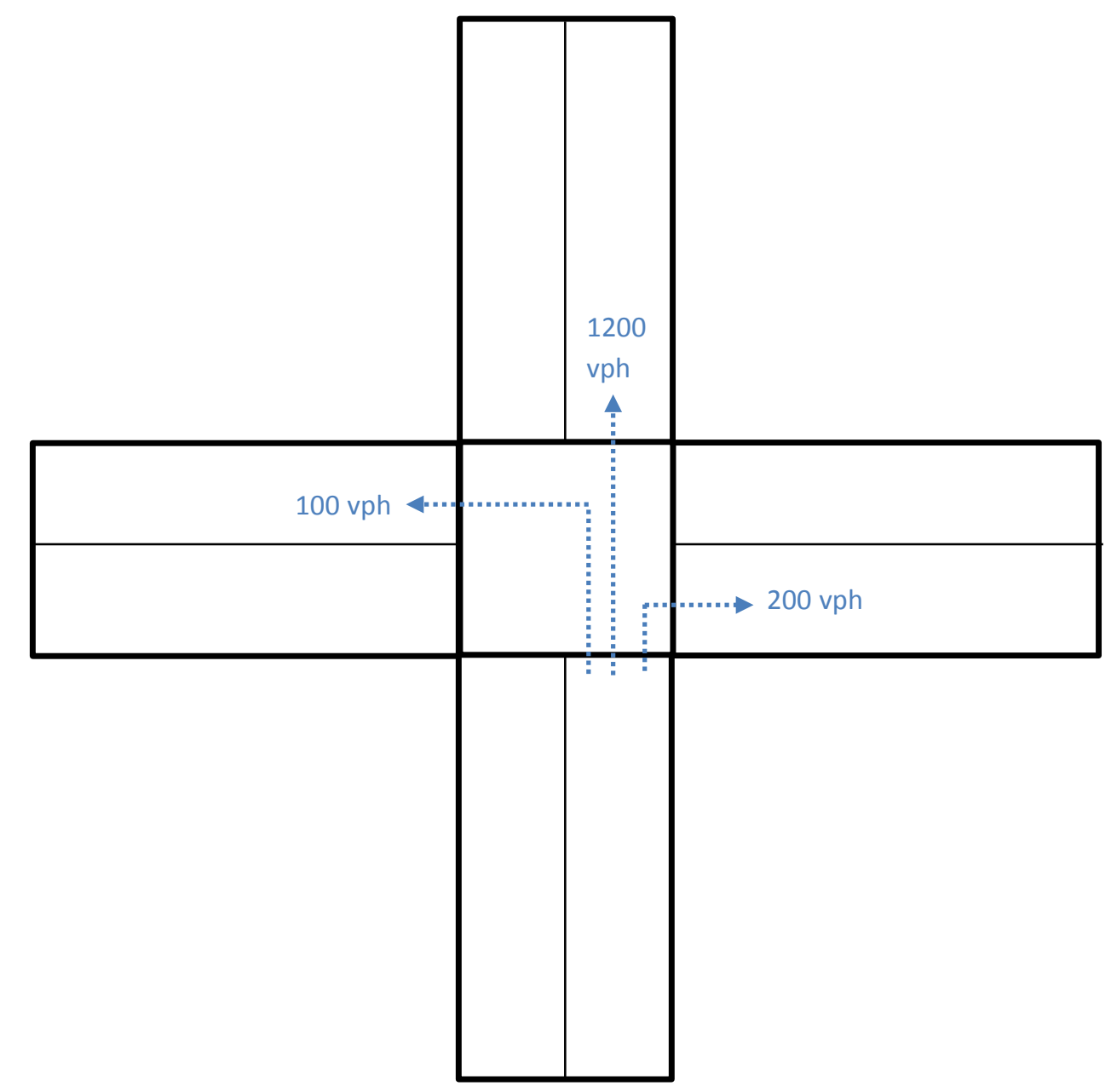

Figure 3: Single intersection case study

a backwards wave speed of half the free flow speed - $30 \mathrm{mi} / \mathrm{hr}(48.3 \mathrm{~km} / \mathrm{hr})$. Capacity and backwards wave speed increase with the proportion of AVs as defined in Section 4.4.

Experiments were performed at 10\% intervals of AV proportion. Each experiment had 1 hour of demand with vehicle departure times randomly chosen. Experiments were repeated 10 times and average travel times per vehicle are shown in Figure 3. Between 0\% and 60\% AVs, average travel time decreases linearly with the proportion of AVs. Between $70 \%$ and $100 \%$ AVs, travel time is almost unchanged. At this point, the capacity of the intersection, increased by reduced headways from AVs, is sufficient for the demand. Slight delays for a few vehicles are observed due to the randomness in the distribution of departure times and of AVs but overall the effect is small. This is consistent with the TBR/LEMITM model: the additional capacity required to reserve all turning movements for $\mathrm{HVs}$ is proportional to the percentage of HVs. In practice, this may be used to predict the intersection delay for arbitrary proportions of AVs and thus determine the point at which TBR/LEMITM improves over signals. Of course, intersection delay also affects intersection demand through 


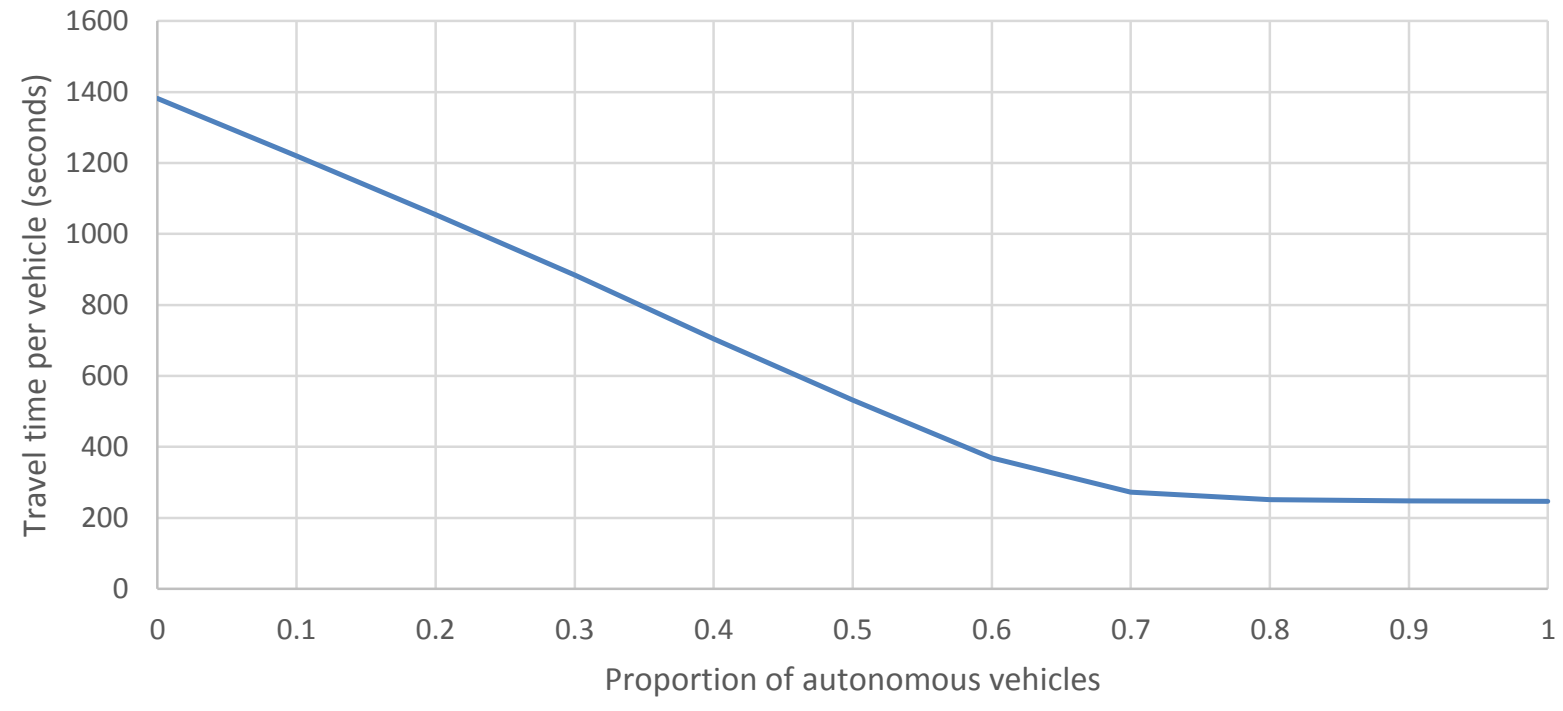

Figure 4: Average travel time per vehicle at different proportions of AVs

\subsection{Shared road dynamic traffic assignment}

We now consider a DTA model using the multiclass CTM and TBR/LEMITM intersection controls to study the predictions of the shared road model with DUE routing. The model was run on the downtown Austin network, which has 171 zones, 546 intersections, 1247 links, and 62836 trips, shown in Figure 5. This network was chosen because many links are arterials or part of the downtown grid and terminate at (currently) signal-controlled intersections. Convergence was measured by comparing travel time with the shortest paths for 15 minute assignment intervals. Let $\tau_{r s t}^{*}$ be the travel time of the shortest path from $r$ to $s$ departing within $t \in \mathscr{T}$, where $\mathscr{T}$ is the set of all assignment intervals. Let $\tau_{v}$ be the travel time of vehicle $v$. The convergence measure of average excess cost (AEC) is then defined as

$$
A E C=\frac{\sum_{(r, s, t) \in Z^{2} \times \mathcal{T}} \sum_{v \in V_{r s t}} \tau_{v}-\tau_{r s t}^{*}}{\sum_{(r, s, t) \in Z^{2} \times \mathcal{T}}\left|V_{r s t}\right|}
$$

where $Z$ is the set of zones and $V_{r s t}$ is the demand from $r$ to $s$ departing within $t \in \mathscr{T}$. DTA used the MSA solution algorithm (see Levin et al., 2014), but more complex techniques could improve convergence. Computation times for 50 iterations of MSA on an Intel Xeon processor running at $3.33 \mathrm{GHz}$ are shown in Figure 6. Since greater proportions of autonomous vehicles increase the network efficiency, and vehicles exit sooner, greater proportions of autonomous vehicles also decrease computation times. The computation times of less than 18 minutes 
per scenario allow a suite of scenarios to be run on the downtown Austin city network within a few hours.

\subsection{Convergence}

Figure 7 shows the average excess cost per iteration for the $50 \%$ AVs scenario. The solution quickly reaches an AEC of less than 50 seconds, but the convergence pattern is slow and non-monotone afterwards. However, that is expected for SBDTA (Levin et al., 2014).

Although convergence is difficult to prove for multiclass formulations even with static traffic assignment (Marcotte \& Wynter, 2004), the multiclass DTA appears to converge to an equilibrium on the downtown Austin city network for all studied proportions of AVs. These results empirically demonstrate that the multiclass dynamic flow and intersection models developed in this paper may be used with DTA on city networks.

\subsection{Travel time predictions}

Total travel time (TTT) with TBR/LEMITM are compared with traffic signals at intersections in Figure 8. DTA was solved for each scenario, so vehicles are considering the average travel times from the correct AV proportion in their route choice. Traffic signals benefit from reduced headways for AVs but delay may be improved by TBR for $100 \%$ AVs (Fajardo et al., 2011). However, this experiment explores the effects of these intersection controls for shared roads. The downtown Austin network (shown in Figure 4) is mostly arterials and downtown grid region. Therefore intersections are the major source of congestion for many links. This is supported by the results: when traffic signals are used, TTT decreases only 


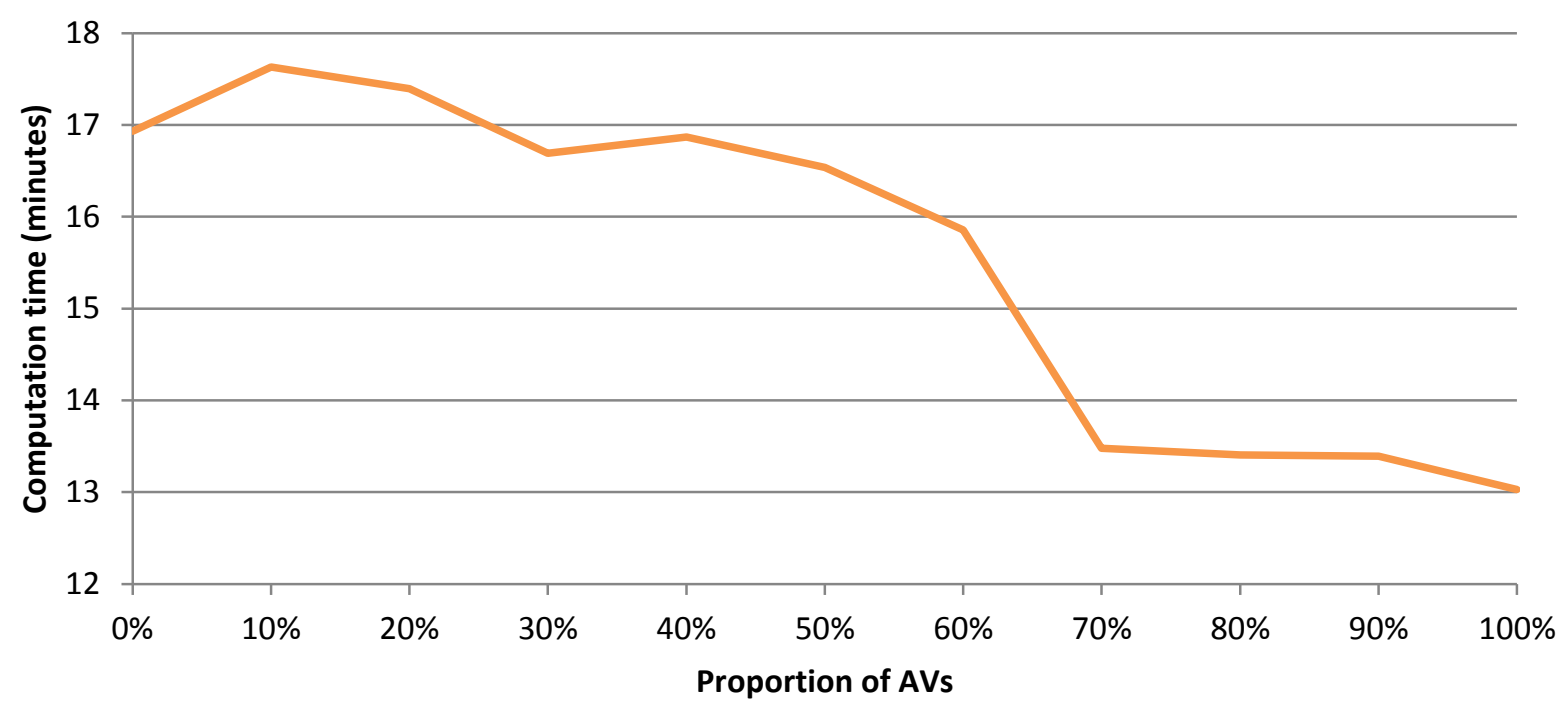

Figure 6: Downtown Austin network

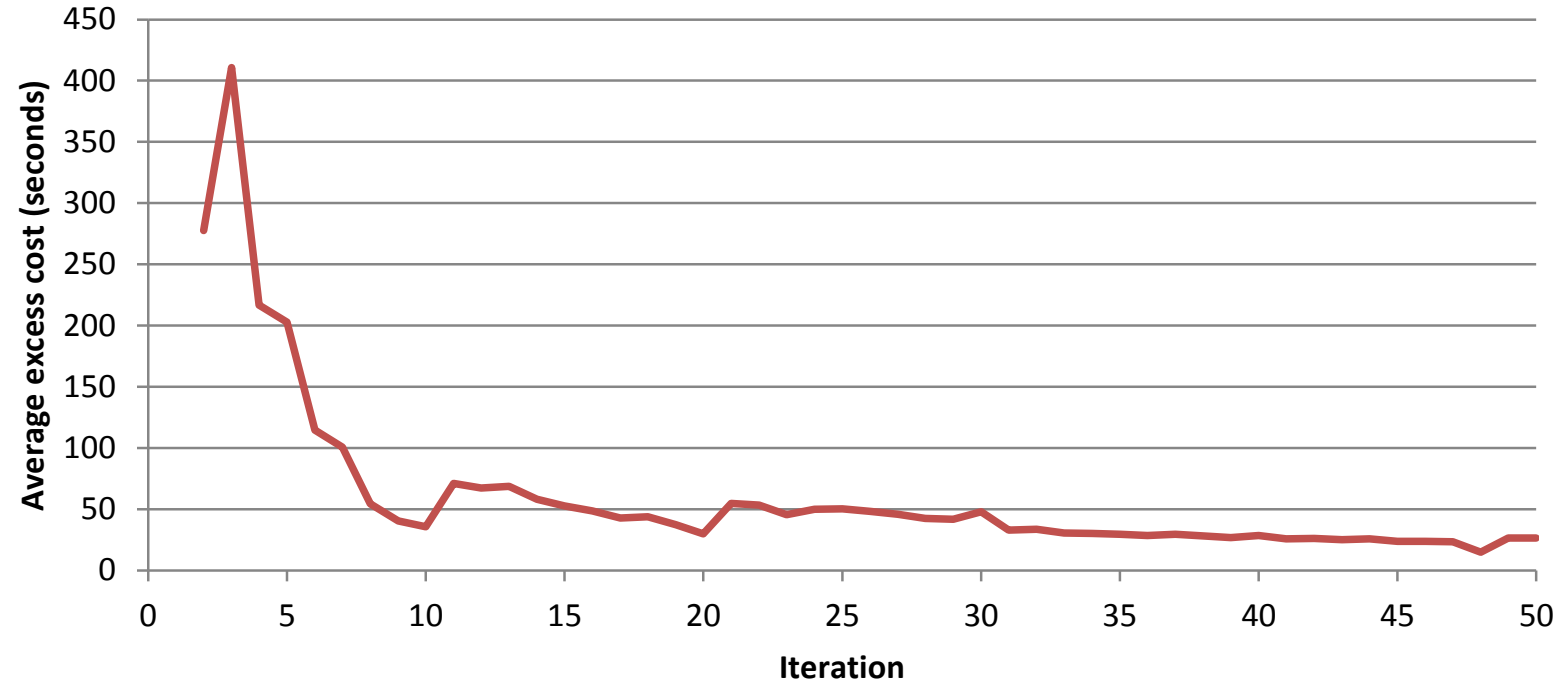

Figure 7: Average excess cost for $50 \%$ AVs scenario 
slightly. Although AVs increase capacity per signal phase, vehicles are still delayed waiting for a phase that allows their turning movement. In contrast, TBR/LEMITM performs much worse when the proportion of AVs is low. For HVs, LEMITM is less efficient than signals because it reserves more of the intersection to ensure safe movement. However, intersection delay and TTT appear to decrease linearly at a significant rate with the proportion of AVs, as with the single intersection results in Section 6.1.

These results suggest that TBR/LEMITM improves over signals after AV penetration reaches around $80 \%$. However, the exact proportion of AVs at which TBR/LEMITM becomes advantageous may vary depending on the city network topology. Note that although Dresner \& Stone (2007) and Bento et al. (2013) study a single shared intersection, route choice may be affected by the proportion of AVs. Intersections with a higher proportion of AVs will experience lower delay and may encourage greater use. Therefore, to estimate the effect of intersection controls on route choice, a DTA framework such as the one presented here should be used.

\section{Conclusions}

Maturing AV technology suggests that AVs will be publicly available within the next few decades. To provide a framework for studying the effects of AVs on city networks, this paper develops a shared road DTA model for human and autonomous vehicles. A multiclass CTM is presented for vehicles traveling at the same speed with capacity and backwards wave speed a function of class proportions. A collision avoidance car following model incorporating vehicle reaction time is used to predict how reduced reaction times might increase capacity and backwards wave speed. These models are generalized to an arbitrary number of classes 
because different AVs may be certified for different reaction times. These models also use continuous flow so that SBDTA models built on continuous flows may incorporate these multiclass predictions.

The second part of a shared road DTA model is the intersection control. We modify the CR model proposed by Levin et al. (2015b) to include the LEMITM reservation model for non-AVs (Bento et al., 2013) while using conventional TBR for AVs. This TBR/LEMITM combination with multiclass CTM flow model is studied in a DTA framework on a single intersection and on a city network. Results verify that use of TBR/LEMITM decreases intersection delay linearly with the proportion of AVs, as is expected from the intersection model. This may be used to predict what AV penetration is required for TBR/LEMITM to improve over traffic signals. Although results on downtown Austin suggest that $80 \%$ AV penetration is required, this may depend on the network topology.

In future work, the capacity and backwards wave speed predicted here should be verified with microsimulation and/or real vehicles. Other such estimations may still be incorporated into the multiclass CTM model presented in this paper. The model of LEMITM should also be calibrated. On a larger scale, determining an efficient shared intersection controls is still an open question to bridge the gap between optimized traffic signals for HVs and TBR for AVs. New shared intersection controls may be implemented in this multiclass framework to study how their performance under DUE routing. This framework might also be used to study the impact of mixed intersection controls (some signals, some TBR/LEMITM) on DUE routing in a traffic network.

\section{Acknowledgements}

The authors gratefully acknowledge the support of the Data-Supported Transportation Operations \& Planning Center and the National Science Foundation under Grant No. 1254921.

\section{References}

Aarts, L., \& Van Schagen, I. (2006). Driving speed and the risk of road crashes: A review. Accident Analysis \& Prevention, 38(2), 215-224.

Bento, L. C., Parafita, R., Santos, S., \& Nunes, U. Intelligent Traffic Management at Intersections: Legacy Mode for Vehicles not Equipped with V2V and V2I Communications. COMMUNICATIONS, 2, V2I.

Brackstone, M., \& McDonald, M. (1999). Car-following: a historical review. Transportation Research Part F: Traffic Psychology and Behaviour, 2(4), 181-196.

Blumberg, M., \& Bar-Gera, H. (2009). Consistent node arrival order in dynamic network loading models. Transportation Research Part B: Methodological, 43(3), 285-300.

Carlino, D., Depinet, M., Khandelwal, P., \& Stone, P. (2012, September). Approximately orchestrated routing and transportation analyzer: Large-scale traffic simulation for autonomous vehicles. In Intelligent Transportation Systems (ITSC), 2012 15th International IEEE Conference on (pp. 334-339). IEEE. 
Carlino, D., Boyles, S. D., \& Stone, P. (2013, October). Auction-based autonomous intersection management. In Proceedings of the 16th IEEE Intelligent Transportation Systems Conference (ITSC).

Chanut, S., \& Buisson, C. (2003). Macroscopic model and its numerical solution for two-flow mixed traffic with different speeds and lengths. Transportation Research Record: Journal of the Transportation Research Board, (1852), 209-219.

Chiu, Y. C., Bottom, J., Mahut, M., Paz, A., Balakrishna, R., Waller, T., \& Hicks, J. (2011). Dynamic traffic assignment: A primer. Transportation Research E-Circular, (E-C153).

Daganzo, C. F. (1994). The cell transmission model: A dynamic representation of highway traffic consistent with the hydrodynamic theory. Transportation Research Part B: Methodological, 28(4), 269-287.

Daganzo, C. F. (1995a). The cell transmission model, part II: network traffic. Transportation Research Part B: Methodological, 29(2), 79-93.

Daganzo, C. F. (1995b). A finite difference approximation of the kinematic wave model of traffic flow. Transportation Research Part B: Methodological, 29(4), 261-276.

Dresner, K., \& Stone, P. (2004, July). Multiagent traffic management: A reservationbased intersection control mechanism. Procgs of 3rd International Joint Conference on Autonomous Agents \& Multiagent Systems-Vol. 2 (pp. 530-537). IEEE Computer Society.

Dresner, K., \& Stone, P. (2006, May). Human-usable and emergency vehicle-aware control policies for autonomous intersection management. In Fourth International Workshop on Agents in Traffic and Transportation (ATT), Hakodate, Japan.

Dresner, K. M., \& Stone, P. (2007, January). Sharing the Road: Autonomous Vehicles Meet Human Drivers. In IJCAI (Vol. 7, pp. 1263-1268).

Fajardo, D., Au, T. C., Waller, S. T., Stone, P., \& Yang, D. (2011). Automated Intersection Control. Transportation Research Record: Journal of the Transportation Research Board, 2259(1), 223-232.

Gartner, N. H., Messer, C. J., \& Rathi, A. K. (2005). Revised monograph on traffic flow theory: a state-of-the-art report. Special Report by the Transportation Research Board of the National Research Council.

Greenshields, B. D., Channing, W., \& Miller, H. (1935). A study of traffic capacity. In Highway research board proceedings (Vol. 1935). National Research Council (USA), Highway Research Board.

Hoogendoorn, S. P., \& Bovy, P. H. (2001). Platoon-based multiclass modeling of multilane traffic flow. Networks and Spatial Economics, 1(1-2), 137-166.

Johansson, G., \& Rumar, K. (1971). Drivers' brake reaction times. Human Factors: The Journal of the Human Factors and Ergonomics Society, 13(1), 23-27.

Kesting, A., Treiber, M., \& Helbing, D. (2010). Enhanced intelligent driver model to access the impact of driving strategies on traffic capacity. Philosophical Transactions of the Royal Society A: Mathematical, Physical and Engineering Sciences, 368(1928), 4585-4605.

Kometani, E., \& Sasaki, T. (1959). Dynamic behaviour of traffic with a nonlinear spacingspeed relationship. In Proceedings of the Symposium on Theory of Traffic Flow, Research 
Laboratories, General Motors (pp. 105-119). New York: Elsevier.

Li, P. Y., \& Shrivastava, A. (2002). Traffic flow stability induced by constant time headway policy for adaptive cruise control vehicles. Transportation Research Part C: Emerging Technologies, 10(4), 275-301.

Levin, M. W., Pool, M., Owens, T., Juri, N. R., \& Waller, S. T. (2014). Improving the Convergence of Simulation-based Dynamic Traffic Assignment Methodologies. Networks and Spatial Economics, 1-22.

Levin, Michael W. \& Stephen D. Boyles (2015a). Effects of Autonomous vehicle ownership on trip, mode, and route choice. Publication pending in Transportation Research Record.

Levin, Michael W. \& Stephen D. Boyles (2015b). Intersection auctions and reservationbased control in dynamic traffic assignment. Publication pending in Transportation Research Record.

Lighthill, M. J., \& Whitham, G. B. (1955). On kinematic waves. II. A theory of traffic flow on long crowded roads. Proceedings of the Royal Society of London. Series A. Mathematical and Physical Sciences, 229(1178), 317-345.

Logghe, S., \& Immers, L. H. (2008). Multi-class kinematic wave theory of traffic flow. Transportation Research Part B: Methodological, 42(6), 523-541.

Marcotte, P., \& Wynter, L. (2004). A new look at the multiclass network equilibrium problem. Transportation Science, 38(3), 282-292.

Marsden, G., McDonald, M., \& Brackstone, M. (2001). Towards an understanding of adaptive cruise control. Transportation Research Part C: Emerging Technologies, 9(1), 3351 .

Miller, Joe. "Google Cars 'designed to Speed"" BBC News. BBC, n.d. Web. 25 Aug. 2014.

Newell, G. F. (1993). A simplified theory of kinematic waves in highway traffic, part I: general theory. Transportation Research Part B: Methodological, 27(4), 281-287.

Newell, G. F. (2002). A simplified car-following theory: a lower order model. Transportation Research Part B: Methodological, 36(3), 195-205.

Nilsson, P., Hussien, O., Chen, Y., Balkan, A., Rungger, M., Ames, A., ... \& Tabuada, P. (2014, December). Preliminary results on correct-by-construction control software synthesis for adaptive cruise control. In Decision and Control (CDC), 2014 IEEE 53rd Annual Conference on (pp. 816-823). IEEE.

Qian, X., Gregoire, J., Moutarde, F., \& De La Fortelle, A. (2014). Autonomous Intersection Management for Mixed Traffic Flow. arXiv preprint arXiv:1407.5813. Richards, P. I. (1956). Shock waves on the highway. Operations research, 4(1), 42-51.

Schakel, W. J., van Arem, B., \& Netten, B. D. (2010, September). Effects of cooperative adaptive cruise control on traffic flow stability. In Intelligent Transportation Systems (ITSC), 2010 13th International IEEE Conference on (pp. 759-764). IEEE.

Schepperle, H., \& Bhm, K. (2007). Agent-based traffic control using auctions. In Cooperative Information Agents XI (pp. 119-133). Springer Berlin Heidelberg.

Tuerprasert, K., \& Aswakul, C. (2010). Multiclass cell transmission model for heteroge- 
neous mobility in general topology of road network. Journal of Intelligent Transportation Systems, 14(2), 68-82.

Van Arem, B., van Driel, C. J., \& Visser, R. (2006). The impact of cooperative adaptive cruise control on traffic-flow characteristics. Intelligent Transportation Systems, IEEE Transactions on, 7(4), 429-436.

van Wageningen-Kessels, F., Van Lint, H., Hoogendoorn, S., \& Vuik, K. (2014). New generic multiclass kinematic wave traffic flow model: Model development and analysis of its properties. Transportation Research Record: Journal of the Transportation Research Board, (2422), 50-60.

Vasirani, M., \& Ossowski, S. (2012). A market-inspired approach for intersection management in urban road traffic networks. Journal of Artificial Intelligence Research, 43(1), 621-659.

Wong, G. C. K., \& Wong, S. C. (2002). A multi-class traffic flow modelan extension of LWR model with heterogeneous drivers. Transportation Research Part A: Policy and Practice, 36(9), 827-841.

Yperman, I., Logghe, S., \& Immers, B. (2005, September). The Link Transmission Model: An efficient implementation of the kinematic wave theory in traffic networks. In Proceedings of the 10th EWGT Meeting, Poznan, Poland. 